\title{
IMPROVING ACCESS TO LIFE-CHANGING IMPLANTS
}

Over 200 dental professionals gathered in London on 20 June for the first DENTSPLY Implants UK and Ireland Symposium. Delegates had the opportunity to learn about new ideas for growing their implant business and improving patient access to this life-changing treatment.

DENTSPLY offers an extensive range of tooth replacement products, endorsed by clinically-documented development spanning three decades. Its portfolio includes implant systems: ANKYLOS, ASTRA TECH Implant System and XiVE, combined with ATLANTIS CAD/CAM digital prosthetics and FRIOS bone augmentation and regeneration material. DENTSPLY Implants has an unrivalled commitment to helping customers develop their skills and treat more implant cases.
A highlight of the programme was the presentation by Dr Marco Degidi on intra-oral welding. The WeldOn system offers a fast and economical method for delivering partial and full-arch immediate restorations. The technique provides a stable, passively-fitting framework for temporary or durable prostheses.

Dr Bernd Giesenhagen, inventor of the bone ring augmentation technique, explained the procedure for implant-supported full arch rehabilitation of the eden-

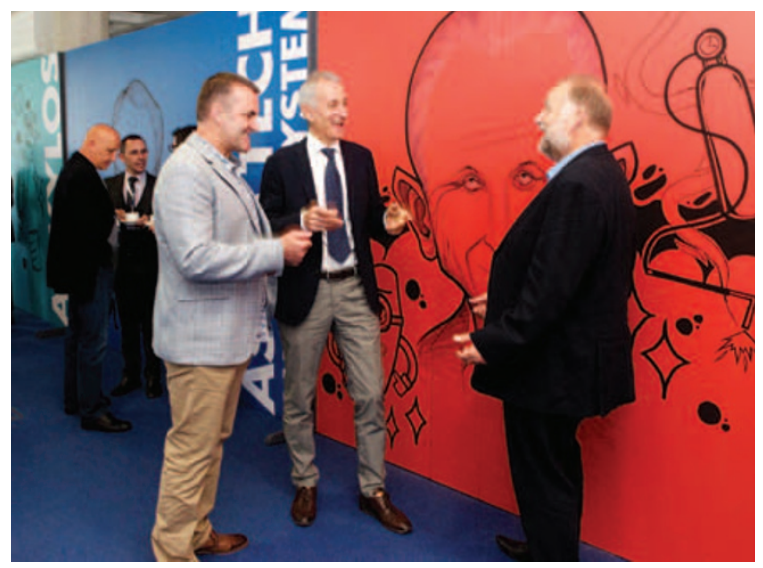

tulous jaw using ANKYLOS Syncone abutments. Dr Clark M. Stanford spoke about his research into the performance and restorative applications of ASTRA TECH Implant System EV.

The latest ideas for hard and soft tissue management in reconstructive implant therapy were presented by Dr Thomas Hanser, a specialist oral surgeon and lecturer on the postgraduate Master of Oral Medicine in Implantology programme at the University of Münster. Presentations by Dr Jan Kielhorn and Mr Ralf Busenbender focused respectively on twenty-first century practice marketing and state-of-theart digital workflow.

For more information on DENTSPLY Implants' products call 0845 4500586 or email implants.uk@ dentsply.com.

\section{ACCESSIBLE 3D IMAGING}

\section{MORE POWER, MORE CONTROL, LESS NOISE}

The noise level of dental turbines stems not only from the operating and drilling noises, but from the spray as well. New Sirona instruments with a fournozzle spray were the best in a comparison of ten turbines from various manufacturers.

Acousticians compared turbines of various manufacturers with respect to acoustic pressure, loudness, and sharpness. Most turbines were significantly louder in the test when they were operated with spray, sometimes up to $10 \mathrm{~dB}(\mathrm{~A})$, which is equivalent to doubling the noise level. The smallest differences in acoustic pressure were found with the two Sirona turbines T1 Control S and T2 Boost S, which were only 2.2 or 0.2 $\mathrm{dB}(\mathrm{A})$ louder when using spray than without.

Sirona introduced a new generation of turbines in 2013 with an innovative four-nozzle spray. Thanks to this, the Sirona turbines were quieter than the other

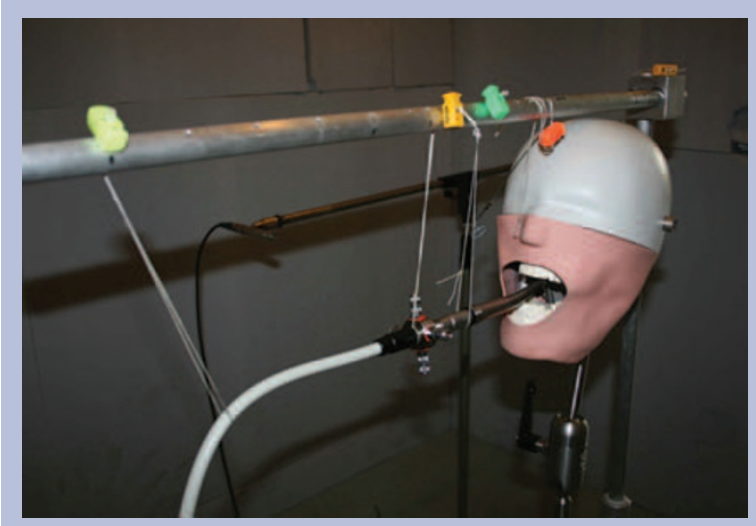

models tested when using spray. In the oral cavity simulation, the acoustic pressure was 60.1 or 61.1 $\mathrm{dB}(\mathrm{A})$. This is equivalent to the noise level during the normal routine in a dental practice. The competitors' products reached levels between 63.5 and $73.8 \mathrm{~dB}(\mathrm{~A})$, some of them exceeding the noise levels caused by cars at a distance of ten metres.

In addition to the greatly reduced noise level, the Sirona turbines in T1 Premium class and T2 Comfort class also have perceptibly more power and better control.

To make it clear how quiet the Sirona turbines are, the company developed a SoundCheck app.

The aim of the game is to arrange drilling noises and sounds in nature according to their noise level. Participants in the game can win Sennheiser HD 600 stereo headphones.

The 'Sirona SoundCheck' is available as a free app via http://appshopper.com/medical/ sirona-soundcheck.

Call Sirona on 08450715040 oremailinfo@sironadental.co.uk.
The Carestream CS8100 3D, available from Henry Schein Dental, effectively blends award-winning and cutting-edge panoramic 2D imaging with 3D capability in a lightweight and ultra-compact design.

The CS8100 3D meets the demands of daily practice by enabling the choice of 2D mode to conduct traditional panoramic exams, and 3D for more advanced applications such as endodontics, implant planning and oral surgery.

The unit's intuitive interface and easy-to-use imaging software is supported by preset programmes and automatic exposure settings, making examinations faster and easier and helping eliminate errors. The unit produces a significantly lower radiation dose than the average CT system and offers four selectable fields of view and fast scanning mode.

Easier positioning, faster image acquisition, and higher image quality makes the CS8100 3D exactly what you need to make more accurate, real-time diagnoses that improve patient care.

www.henryschein.co.uk 